\title{
A BANACH SPACE ON WHICH THERE ARE FEW OPERATORS
}

\section{SAHARON SHELAH AND JURIS STEPRĀNS}

(Communicated by John B. Conway)

\begin{abstract}
It is shown that there is a nonseparable Banach space on which every operator has the form $S+\rho I$ where $\rho$ is a scalar and $S$ is an operator with separable range.
\end{abstract}

An open problem in the theory of Banach spaces is the construction of a Banach space with the property that all operators from it to itself are the sum of a multiple of the identity and a compact operator. Since compact operators have separable range a related question is to find a (nonseparable) Banach space on which all operators are the sum of a multiple of the identity and an operator with separable range. This was first done by Shelah using an extra set-theoretic axiom in [1]. It is the purpose of this paper, which is the continuation of research which began in [3], to prove the same result without appealing to axioms other than those of ZFC.

The following notation will be used. The integers will be considered as ordinals so that $n \in m$ means the same as $n<m$. The set of integers is denoted as $\omega$ instead of $\mathbf{N}$ and the first uncountable ordinal is $\omega_{1}$. The symbols $[X]^{\lambda}$ and $[X]^{<\lambda}$ denote, respectively, the set of $\lambda$ sized subsets of $X$ and less than $\lambda$ sized subsets of $X$. The set of functions from $Y$ to $X$ is denoted ${ }^{Y} X$. In particular, ${ }^{\omega_{1}} \mathbf{R}$, when endowed with the usual pointwise operations, is the real vector space of " $\omega_{1}$-tuples". For $X \subseteq \omega_{1}$ the characteristic function of $X$ will be denoted by $\mathbf{1}_{X}$.

Let us begin by reviewing the construction from [2] of a Banach space on which every operator is the sum of an operator with separable range and one which is diagonal with respect to a fixed basis. Let $\mathfrak{A} \subseteq\left[\omega_{1}\right]^{<\kappa_{0}}$ be such that:

1. $\bigcup \mathfrak{A}=\omega_{1}$.

2. $\mathfrak{A}$ is closed under subsets.

It is possible to define a function \|\|$_{\mathfrak{A}}$ on ${ }^{\omega_{1}} \mathbf{R}$ by

$$
\|f\|_{\mathfrak{A}}=\sup \left\{\sum\{|f(\alpha)| ; \alpha \in A\} ; A \in \mathfrak{A}\right\} .
$$

Let $B=\left\{{ }^{\omega_{1}} \mathbf{R} ;\|f\|_{\mathfrak{A}}\right.$ is finite $\}$ and note that $\left(B,\|\|_{\mathfrak{A}}\right)$ is a normed linear space. Of course if $\mathfrak{A}=\left[\omega_{1}\right]^{<\omega}$ then $B=l_{1}\left(\omega_{1}\right)$ but we will assume that there is a family $\mathfrak{B} \subseteq\left[\omega_{1}\right]^{<\omega}$ such that:

3. $\mathfrak{B}$ is closed under subsets.

4. $\mathfrak{A} \cap \mathfrak{B} \subseteq\left[\omega_{1}\right]^{1}$.

5. For all families of disjoint pairs $\left\{\{a(\alpha), b(\alpha)\} ; \alpha \in \omega_{1}\right\}$ and integers $k$ there is $X \in\left[\omega_{1}\right]^{k}$ such that $\{a(\alpha) ; \alpha \in X\} \in \mathfrak{A}$ and $\{b(\alpha) ; \alpha \in X\} \in \mathfrak{B}$.

Received by the editors March 3, 1986 and, in revised form, July 23, 1987.

1980 Mathematics Subject Classification (1985 Revision). Primary 46B20; Secondary 04A05.

Key words and phrases. Nonseparable Banach space, operator with separable range, uncountable cardinal, partition relation.

(C) 1988 American Mathematical Society $0002-9939 / 88 \$ 1.00+\$ .25$ per page 
LEMMA 1. Let $\nu$ be a seminorm on $B$ and let $B_{\xi}$ be the closure of $\left\{\mathbf{1}_{\{\alpha\}} ; \alpha \in \xi\right\}$ in $B$ with respect to the norm $\nu+\|\|_{\mathfrak{A}}$. Suppose that

6. there is $M$ such that $\nu\left(\mathbf{1}_{b}\right) \leq M$ for all $b \in \mathfrak{B}$,

7. the projection mapping $\pi: B_{\omega_{1}} \rightarrow B_{\xi}$ is bounded for each $\xi \in \omega_{1}$.

Then every operator from $B_{\omega_{1}}$ to $B_{\omega_{1}}$ is the sum of a separable operator and one which is diagonal with respect to the basis $\left\{\mathbf{1}_{\{\alpha\}} ; \alpha \in \omega_{1}\right\}$.

PROOF. To see this suppose that $T: B_{\omega_{1}} \rightarrow B_{\omega_{1}}$ is a bounded operator which is not of the desired form. First note that there is a family of pairs $\{\{a(\eta), b(\eta)\}$; $\left.\eta \in \omega_{1}\right\}$ such that

8. $a(\eta) \neq b(\eta)$ for $\eta \in \omega_{1}$,

9. $T\left(1_{\{b(\eta)\}}\right)(a(\eta)) \neq 0$ for $\eta \in \omega_{1}$,

10. if $\eta \neq \varsigma$ then $\{a(\eta), b(\eta)\} \cap\{a(\eta), b(\eta)\}=\varnothing$.

To see why this is so note that otherwise there is some $\xi$ such that $T\left(\mathbf{1}_{\{\eta\}}\right)=$ $r_{\eta} \mathbf{1}_{\{\eta\}}+f_{\eta}$ where $f_{\eta} \in B_{\xi}$ for all $\eta \geq \xi$. But this implies that $T=D+T \circ \pi_{\xi}$ where $D\left(\mathbf{1}_{\eta}\right)=r_{\eta} \mathbf{1}_{\eta}$. Since $\pi_{\xi}$ is assumed to be bounded it follows that $T$ is of the desired form.

Now notice that the family of pairs may be thinned out so that $T\left(\mathbf{1}_{\{b(\eta)\}}\right)(a(\beta))$ $=0$ if $\eta \neq \beta$. The reason is that otherwise there is an uncountable set, $Y$, and $\beta_{0}$ such that $T\left(\mathbf{1}_{\{b(\eta)\}}\right)\left(a\left(\beta_{0}\right)\right) \neq 0$ for all $\eta \in Y$. Without loss of generality $T\left(\mathbf{1}_{\{b(\eta)\}}\right)\left(a\left(\beta_{0}\right)\right) \geq \delta$ for $\eta \in Y$. Let $n \delta \geq M\|T\|$. An application of (5) yields $X \in[Y]^{n}$ such that $\{b(\eta) ; \eta \in X\} \in \mathfrak{B}$ and hence $\nu\left(\mathbf{1}_{\{b(\eta) ; \eta \in X\}}\right) \leq M$ while $\nu\left(T\left(\mathbf{1}_{\{b(\eta) ; \eta \in X\}}\right)\right) \geq\left\|T\left(\mathbf{1}_{\{b(\eta) ; \eta \in X\}}\right)\right\|_{\mathfrak{A}} \geq n \delta \geq M\|T\|$.

It may also be assumed that there is $\rho>0$ such that $T\left(\mathbf{1}_{\{b(\eta)\}}\right)(a(\eta))>\rho$ for $\eta \in \omega_{1}$. Choose $k$ such that $k \rho \geq M\|T\|$. Now use (5) to find $X \in\left[\omega_{1}\right]^{k}$ such that $\{b(\eta) ; \eta \in X\} \in \mathfrak{A}$ and $\{b(\eta) ; \eta \in X\} \in \mathfrak{B}$. It follows that $\nu\left(\mathbf{1}_{\{b(\eta) ; \eta \in X\}}\right) \leq M$ while $T\left(\mathbf{1}_{\{b(\eta)\}}\right)(a(\eta)) \geq \nu\left(\mathbf{1}_{\{a(\eta) ; \eta \in X\}}\right) \geq k \rho \geq M\|T\|$.

A seminorm on $B$ will now be constructed which satisfies (6) and (7) as well as some additional properties. In order to define this seminorm another family of finite sets with certain properties will have to be hypothesized. Let $\mathfrak{D}$ be a subset of $\left[\left[\omega_{1}\right]^{2}\right]^{<\omega}$ satisfying the following properties:

11. id $D \in \mathfrak{D}$ then $D=\{\{a(j), b(j)\} ; j \in k\}$ for some $k$ and $\max \{a(j), b(j)\}<$ $\min \{a(j+1), b(j+1)\}$ for $j \in k-1$,

12. if $A \in \mathfrak{A}, B \in \mathfrak{B}$ and $D \in \mathfrak{D}$ then $|A \cap(\bigcup D)| \leq 2$ and $|B \cap(\bigcup D)| \leq 2$,

13. if $D$ and $D^{\prime}$ are in $\mathfrak{D}$ then

$$
\mid\left\{d \in D ;\left(\exists d^{\prime} \in D^{\prime}\right)\left(d \neq d \cap d^{\prime} \neq \varnothing\right\} \mid \leq 5,\right.
$$

14. if $Z \subseteq\left[\omega_{1}\right]^{2}$ consists of disjoint pairs and $k \in \omega$ then $\varnothing \neq[Z]^{k} \cap \mathfrak{D}$.

The seminorm $\nu$ can now be defined from the family $\mathfrak{D}$. If $f \in{ }^{\omega_{1}} \mathbf{R}$ then $\nu(f)=\sup \left\{\sum\{|f(\alpha)-f(\beta)| ;\{\alpha, \beta\} \in D\} ; D \in \mathfrak{D}\right\}$. It must be shown that $\nu$ satisfies (6) and (7). It follows directly from (12) that $\nu\left(\mathbf{1}_{b}\right) \leq 2$ for $b \in \mathfrak{B}$. To see that (7) holds it suffices to show that $\nu\left(\pi_{\xi}(f)\right) \leq 2$ provided that $f \in{ }^{\omega_{1}} \mathbf{R}$, $\nu(f)+\|f\|_{\mathfrak{A}} \leq 1$ and $\xi \in \omega_{1}$. But if $D \in \mathfrak{D}$ then there is at most one $d \in D$ such that $d \neq d \cap \xi \neq \varnothing$ and hence $\sum\left\{\left|\pi_{\xi}(f)(\alpha)-\pi_{\xi}(f)(\beta)\right| ;\{\alpha, \beta\} \in D\right\} \leq 2$.

It remains to be shown that if $T: B_{\omega_{1}} \rightarrow B_{\omega_{1}}$ is a bounded operator then $T=\sigma I+S$ where $\sigma$ is a scalar and $S$ has separable range. The following lemma will be used in establishing this. 
LEMMA 2. If $D \in \mathfrak{D}$ then $\nu\left(\mathbf{1}_{\cup D}\right) \leq 5$.

PROOF. Notice that

$$
\begin{aligned}
& \sum\left\{\left|\mathbf{1}_{\cup D}(\alpha)-\mathbf{1}_{\cup D}(\beta)\right| ;\{\alpha, \beta\} \in D^{\prime}\right\} \\
& \quad=\left|\left\{d^{\prime} \in D^{\prime} ;(\exists d \in D)\left(\varnothing \neq d \cap d^{\prime} \neq d\right)\right\}\right|
\end{aligned}
$$

For each $D^{\prime} \in \mathfrak{D}$. It follows directly from (13) that $\nu\left(\mathbf{1}_{\cup D}\right) \leq 5$.

THEOREM. If $B_{\omega_{1}}$ is the closure in $B$ of $\left\{\mathbf{1}_{\{\eta\}} ; \eta \in \omega_{1}\right\}$ with respect to the norm $\nu+\|\|_{\mathfrak{A}}$ then every operator on $B_{\omega_{1}}$ is the sum of a multiple of the identity and an operator with separable range.

PROOF. Let $T: B_{\omega_{1}} \rightarrow B_{\omega_{1}}$ be a bounded operator. Since Lemma 1 already implies that $T$ is the sum of a diagonal operator with respect to the basis $\left\{\mathbf{1}_{\{\eta\}}\right.$; $\left.\eta \in \omega_{1}\right\}$ it may as well be assumed that $T$ is diagonal. If $T$ is not a multiple of the identity then it is possible to choose disjoint pairs $\left\{\{a(\eta), b(\eta)\} ; \eta \in \omega_{1}\right\}$ and $\rho>0$ such that $\left|T\left(\mathbf{1}_{\cup D}\right)(a(\eta))-T\left(\mathbf{1}_{\cup D}\right)(b(\eta))\right| \geq \rho$ for each $\eta \in \omega_{1}$. Let $k \rho \geq 5\|T\|$. Now use (14) to find $X \in\left[\omega_{\mathbf{1}}\right]^{k}$ such that $D=\{\{a(\eta), b(\eta)\} ; \eta \in X\} \in \mathfrak{D}$. From Lemma 2 it follows that $\nu\left(\mathbf{1}_{\cup D}\right)+\left\|\mathbf{1}_{\cup D}\right\|_{\mathfrak{A}} \leq 5$ while, on the other hand,

$$
\nu\left(T\left(\mathbf{1}_{\cup D}\right)\right) \geq \sum\left\{\left|T\left(\mathbf{1}_{\cup D}\right)(a(\eta))-T\left(\mathbf{1}_{\cup D}\right)(b(\eta))\right| ; \eta \in X\right\}>\rho|X| \geq 5\|T\| .
$$

All that remains to be shown is that it is possible to find families $\mathfrak{A}, \mathfrak{B}$ and $\mathfrak{D}$ satisfying properties (1)-(5) and (11)-(14). To this end let us recall the following fact from [2].

15. For any set $I$ of cardinality $\omega_{1}$ there is a function $F:\left[\omega_{1}\right]^{2} \rightarrow I$ satisfying the following property: if $\{\{a(\eta), b(\eta)\} ; \eta \in \omega\} \subseteq\left[\omega_{1}\right]^{2}$ is a collection of disjoint pairs and $i \in I$ then there is $\{\eta, \gamma\} \in\left[\omega_{1}\right]^{2}$ such that $\{a(\eta), a(\gamma)\} \in F^{-1}\{i\}$ and $\{b(\eta), b(\gamma)\} \in F^{-1}\{i\}$. First note that any function satisfying (15) also satisfies the following, seemingly stronger condition: If $\left\{\{a(\eta), b(\eta)\} ; \eta \in \omega_{1}\right\} \subseteq\left[\omega_{1}\right]^{2}$ is a collection of disjoint pairs, $k \in \omega$ and $i \in I$ then there is $\Xi \in\left[\omega_{1}\right]^{k}$ such that $\{a(\eta), a(\gamma)\} \in F^{-1}\{i\}$ and $\{b(\eta), b(\gamma)\} \in F^{-1}\{i\}$ for each $\{\eta, \gamma\} \in[\Xi]^{2}$. This follows directly from the partition relation $\omega_{1} \rightarrow\left(\omega_{1}, \omega\right)^{2}$.

To construct $\mathfrak{A}, \mathfrak{B}$ and $\mathfrak{D}$ let $F:\left[\omega_{1}\right]^{2} \rightarrow 3 \times\left[\omega_{1}\right]^{<\omega}$ satisfy the condition stated in (15) and let $F_{0}$ and $F_{1}$ be the coordinate functions of $f$ so that $F=F_{0} \times F_{1}$. Then let

$$
\mathfrak{A}=\left\{A \in\left[\omega_{1}\right]^{<\omega} ;[A]^{2} \subseteq F_{0}^{-1}\{0\}\right\}
$$

and

$$
\mathfrak{B}=\left\{B \in\left[\omega_{1}\right]^{<\omega} ;[B]^{2} \subseteq F_{0}^{-1}\{1\}\right\} .
$$

It is easy to see that properties (1)-(4) are satisfied and (5) follows from a straightforward application of the remarks following (15). Define $\mathfrak{D}$ to consist of all $D$ such that $D=\{\{a(j), b(j)\} ; j \in k\}$ for some $k$ and the following properties hold:

16. $\max \{a(j), b(j)\}<\min \{a(j+1), b(j+1)\}$ for $j \in k-1$.

17. $[\{a(j) ; j \in k\}]^{2} \cup[\{b(j) ; j \in k\}]^{2} \subseteq F_{0}^{-1}\{2\}$.

18. $F_{1}(\{a(i), a(j)\})=F_{1}(\{b(i), b(j)\})=\bigcup\{\{a(m), b(m)\} ; m \in \min \{i, j\}\}$.

Property (16) ensures that (11) will be satisfied while (17) takes care of (12). To see that (13) is satisfied let $\left\{D, D^{\prime}\right\} \in[\mathfrak{D}]^{2}$ and let $D=\{\{a(j), b(j)\} ; j \in k\}$ and $D^{\prime}=\left\{\left\{a^{\prime}(j), b^{\prime}(j)\right\} ; j \in k^{\prime}\right\}$. If $(13)$ is not satisfied then let $C \subseteq k^{\prime}$ be the 
five largest values such that if $j \in C$ then $\varnothing \neq \bigcup D \cap\left\{a^{\prime}(j), b^{\prime}(j)\right\}$. Since either $a^{\prime}(j) \in \bigcup D$ or $b^{\prime}(j) \in \bigcup D$ for each $j \in C$ it follows that there is $C^{\prime} \in[C]^{3}$ such that (without loss of generality) $a^{\prime}(j) \in \bigcup D$ for $j \in C^{\prime}$. For each $j \in C^{\prime}$ either $a^{\prime}(j)=b(i) \in \bigcup D$ or $a^{\prime}(j)=a(i) \in \bigcup D$ for some $i \in k$. Again there must be $\left\{j, j^{\prime}\right\} \in\left[C^{\prime}\right]^{2}$ such that the same choice is made and it may again be assumed (without loss of generality) that there are $i$ and $i^{\prime}$ such that $a^{\prime}(j)=b(i)$ and $a^{\prime}\left(j^{\prime}\right)=b\left(i^{\prime}\right)$. It follows from (18) that $\bigcup\left\{\left\{a^{\prime}(m), b^{\prime}(m)\right\} ; m<\min \left\{j^{\prime}, j\right\}\right\}$

$$
F_{1}\left(\left\{a^{\prime}(j), a^{\prime}\left(j^{\prime}\right)\right\}\right)=F_{1}\left(\left\{b(i), b\left(i^{\prime}\right)\right\}\right)=\bigcup\left\{\{a(m), b(m)\} ; m<\min \left\{i^{\prime}, i\right\}\right\} .
$$

It follows from (16) that if $m<\min \left\{j, j^{\prime}\right\}$ then $\left\{a^{\prime}(m), b^{\prime}(m)\right\}=\{a(m), b(m)\}$ and hence (13) is satisfied.

Finally it must be shown that (14) holds. Let $\left\{\left\{a(\eta), b(\eta) ; \eta \in \omega_{1}\right\} \subseteq\left[\omega_{1}\right]^{2}\right.$ be a collection of disjoint pairs. It suffices to show that

19. if $X \in\left[\omega_{1}\right]^{<\omega}$ and $Y \in\left[\omega_{1}\right]^{\omega_{1}}$ are such that $\{\{a(\eta), b(\eta) ; \eta \in X \cup\{\vartheta\}\}$ satisfies (18) for each $\vartheta \in Y$ then it is possible to choose $\vartheta_{0} \in Y$ and $Y^{\prime} \in[Y]^{\omega_{1}}$ such that $\left\{\{a(\eta), b(\eta)\} ; \eta \in X \cup\left\{\vartheta_{0}\right\} \cup\{\vartheta\}\right\}$ satisfies (18) for each $\vartheta \in Y^{\prime}$.

The reason is that then (18) can be satisfied by a recursive construction. Therefore suppose that (19) is false. Then for each $\vartheta \in \omega_{1}$ there are no more than countably many $\tau \in \omega_{1}$ such that $\{\{a(\eta), b(\eta)\} ; \eta \in X \cup\{\vartheta\} \cup\{\tau\}\}$ satisfies (18). Note also that, because of the hypothesis on $X$ and $Y$, it must be the case that the failure of $(18)$ is because either

$$
F_{1}(\{a(\vartheta), a(\tau)\}) \neq \bigcup\{\{a(\eta), b(\eta)\} ; \eta \in X\}
$$

or

$$
F_{1}(\{b(\vartheta), b(\tau)\}) \neq \bigcup\{\{a(\eta), b(\eta)\} ; \eta \in X\}
$$

Now by thinning out $Y$ it is possible to find $Z \in[Y]^{\omega_{1}}$ such that if $\{\vartheta, \tau\} \in[Z]^{2}$ then either

$$
F_{1}(\{a(\vartheta), a(\tau)\}) \neq \bigcup\{\{a(\eta), b(\eta)\} ; \eta \in X\}
$$

or

$$
F_{1}(\{b(\vartheta), b(\tau)\}) \neq \bigcup\{\{a(\eta), b(\eta)\} ; \eta \in X\}
$$

which contradicts (15).

It should be remarked that credit for the relative simplicity of the construction just presented, and isolating the role of the combinatorial principle (15) in particular, should be given to the referee. This combinatorial principle is very similar to the statement that $\omega_{1} \rightarrow\left[\omega_{1}\right]_{\omega_{1}}^{2}$ is false, which was proved by Todorcevic [4]. The latter simply says that it is possible to colour the edges of the complete graph on $\aleph_{1}$ vertices in such a way that each colour will appear among the edges determined by any subset of the vertices of size $\aleph_{1}$. The strengthening achieved in (15) is that now given any two subsets of size $\aleph_{1}$ (and an indexing of both) each colour will appear simultaneously on corresponding (with respect to the indexing) edges. It might be interesting to try to construct a Banach space with few operators directly from the negation of $\omega_{1} \rightarrow\left[\omega_{1}\right]_{\omega_{1}}^{2}$. 


\section{REFERENCES}

1. S. Shelah, A Banach space with few operators, Israel J. Math. 30 (1978), 181-191.

2. __ A graph which embeds all small graphs on any large set of vertices, preprint.

3. S. Shelah and J. Steprāns, Extraspecial p-groups, Ann. Pure Appl. Logic 34 (1987), 87-97.

4. S. Todorcevic, Partitioning pairs of countable ordinals, Acta Math. 159 (1987), 261-294.

Department of MAThematics, Hebrew UNiversity, JeRusalem, ISRAEL

DEPARTMENT OF MATHEMATICS, YORK UNIVERSity, DOWNSVIEW ONTARIO M3J 1P3, CANADA 